\title{
Considering the Effect of a Ligand as New Complexing Agent in the Characteristics of $\mathrm{TiO}_{2}$ Nanoparticles
}

\author{
Tahereh Gholami ${ }^{\text {a }}$, Mehdi Bazarganipour ${ }^{\mathrm{b}}$, Masoud Salavati-Niasari*a ${ }^{\mathrm{a}}$, Nooshin Mir ${ }^{\mathrm{c}}$, Masood \\ Hamadanian $^{\text {a }}$, Samira Bagheri ${ }^{\mathrm{d}}$
}

${ }^{a}$ Institute of Nano Science and Nano Technology, University of Kashan, Kashan, P. O. Box. 87317-51167, I. R. Iran.

${ }^{b}$ Nanotechnology and Advanced Materials Institute, Isfahan University of Technology, Isfahan, 84156-83111, I. R. Iran.

${ }^{c}$ Department of Chemistry, University of Zabol, P. O. Box 98615-538, Zabol, Islamic Republic of Iran ${ }^{d}$ Centre for Research in Nanotechnology \& Catalysis (NANOCAT); 3rd Floor, Block A, Institute of Postgraduate Studies (IPS) building; University of Malaya, Kuala Lumpur-50603, MALAYSIA

*Corresponding authors. Tel. +98 31 55912383; Fax +98 31 55913201. E-mail address: $\underline{\text { salavati@kashanu.ac.ir }}$

\section{Abstract}

The effect of a Schiff-base ligand N,N'-bis[(Z)-(2-nitrophenyl)methylidene]propane-1,3-diamine; (Salpn( $\left.\left.\mathrm{NO}_{2}\right) 2\right)$ ) on the size and optical properties and the effect of time in preparation of $\mathrm{TiO}_{2}$ nanoparticles in a two-step sol-gel method were investigated. Different amounts of Schiff-base ligand were applied and the as-prepared products were characterized using X-ray diffraction (XRD), scanning electron microscopy (SEM), Fourier transform infrared (FT-IR) spectrum, Electron Dispersive X-ray spectroscopy (EDX) and ultraviolet-visible (UV-Vis).

KEYWORDS: Colloidal solutions; Sol-gel chemistry; Chemical synthesis; Optical properties; Electron microscopy; Aqueous solutions. 


\section{Introduction}

$\mathrm{TiO}_{2}$ nanoparticles are widely utilized as photocatalysts, ceramic materials, fillers, coatings, pigments, cosmetics, etc. For these purposes, it is strongly desirable that the $\mathrm{TiO}_{2}$ nanoparticles to be uniform and completely controlled in size, shape, and crystal structure.

A broad diversity of procedures has been developed for the preparation of $\mathrm{TiO}_{2}$ nanoparticles, until now. Sol-gel [1, 2], reverse micelle [3], sonochemical [4], microwave [5] and hydrothermal [6] are some examples of chemical routes for synthesis of well-define $\mathrm{TiO}_{2}$ nanoparticles. Two-step sol-gel procedure is a similar adopted method of conventional "gel-sol" method, developed by Sugimoto [7-10].

A complexing agent assisted sol-gel procedure utilizing an organic ligand as a modulator can tailor the crystal structure and optical properties of thin films such as $\mathrm{TiO}_{2}[11,12]$. Surely, not each molecule is an equally good ligand. Fundamentally, ligands that accomplish the task of adsorbent and stabilizer have a functional head group and one or more hydrocarbon large groups, and both elements perform a role in the control of nucleation and growth [13-21].

In this research, $\mathrm{TiO}_{2}$ nanoparticles were prepared utilizing organic Schiff-base ligand as complexing agents by a two step sol-gel procedure. The intension of this research is investigating the role of Schiff-base ligand on the size of the $\mathrm{TiO}_{2}$ nanoparticles. As regards, to our knowledge there is not any report on the effect of this organic Schiff-base ligand on the size and properties of $\mathrm{TiO}_{2}$ nanoparticles.

\section{Experimental}

\subsection{Materials and characterization}

Titanium(IV) ethoxide, and triethanolamine(TEOA) used in our experiments were purchased from Merck. For characterization of the products X-ray diffraction (XRD) patterns were recorded by a Rigaku D-max C III, X-ray diffractometer using Ni-filtered $\mathrm{Cu}$ Ka radiation. GC-2550TG (Teif Gostar Faraz Company, Iran) were used for all chemical analyses. Scanning electron microscopy (SEM) images were obtained on Philips XL-30ESEM. 
Fourier transform infrared (FT-IR) spectra were recorded on Shimadzu Varian 4300 spectrophotometer in $\mathrm{KBr}$ pellets. Optical analyses were performed using a V-670 UV-VIS-NIR Spectrophotometer (Jasco).

\subsection{Preparation of $N_{,} N^{\prime}$-bis[(Z)-(2-nitrophenyl)methylidene]propane-1,3-diamine; $\operatorname{Salpn}\left(\mathrm{NO}_{222}\right.$ [22]}

The symmetrical Schiff-base ligand, N,N'-bis[(Z)-(2-nitrophenyl)methylidene]propane-1,3- diamine, $\mathrm{C}_{17} \mathrm{H}_{16} \mathrm{~N}_{4} \mathrm{O}_{4}\left(\mathrm{Salpn}-\mathrm{NO}_{2}\right)_{2}$, was prepared according to previously published methods, by refluxing 0.05 mol of 2nitrobenzaldehyde (Merck-Schuchard, purified by distillation) and $0.025 \mathrm{~mol}$ of propylenediamine (Aldrich Chemie, 99\%, purified by distillation) in $30 \mathrm{ml}$ of dried ethanol, for $30 \mathrm{~min}$ and cooling the reaction mixture. The Schiff-base ligand was separated as yellow powder and was recrystallized twice from methanol (yield 90\%). The compound was stable at room temperature and was characterized by FT-IR spectroscopy.

\subsection{Preparationof $\mathrm{TiO}_{2}$ nanoparticles}

The standard procedure for the preparation of $\mathrm{TiO}_{2}$ nanoparticles was conducted according to the literature [2328]. Stock solution of $\mathrm{Ti}^{4+}(0.5 \mathrm{M})$ which is stable against hydrolysis at room temperature was prepared by mixing titanium(IV) ethoxide (TEO) and TEOA with a molar ratio of TEO:TEOA=1:2, followed by the addition of distilled water. The final $\mathrm{pH}$ of solutions was 9.6 and was fixed at this $\mathrm{pH}$ by mixing with $\mathrm{HClO}_{4}$ or $\mathrm{NaOH}$ solution after addition of complexing agents. Salpn $\left(\mathrm{NO}_{2}\right)_{2}$ ligand with different ratios to the $\mathrm{Ti}^{4+}$ precursor(Table 1)was further added as complexing agents to each solution. The solution was placed in a Teflon-lined autoclave and aged at $100{ }^{\circ} \mathrm{C}$ for $24 \mathrm{~h}$ for gelation; then, the temperature was increased to $140{ }^{\circ} \mathrm{C}$ for $72 \mathrm{~h}$ to nucleate and grow $\mathrm{TiO}_{2}$ nanocrystals. $\mathrm{TiO}_{2}$ nanoparticles were prepared in the different times (Table 2). The resulting sol was washed with and centrifuged from $\mathrm{NaOH}$ (six times), $\mathrm{HNO}_{3}$ (two times), and distilled water (four times) to remove residual organic compounds from the surface of the nanoparticles.

\section{Results and discussion}

The X-ray diffraction patterns of as-prepared product obtained from the two-step reaction have been shown in

Fig. 1. Fig. 1a-c show the XRD patterns of samples S1, S0 and S1T0 which have been prepared in presence of 
both ligand and TEOA, in absence of ligand, and in absence of TEOA, respectively. All diffraction peaks can be well indexed to pure anatase structural titanium dioxide. All the peaks were labelled and can be indexed to JCPDS Card No. 76-1935, 04-0477, for sample S1, S0 and S1T0, respectively. The XRD patterns of samples show different line broadening. Generally, the crystalline domain size decreases with increasing line broadening. The line broadening of the peak of the (101) index is related to the size of the crystalline phase. The data for the full width at half maximum (FWHM) of the samples S1, S0 and S1T0 at $2 \theta=25.35,25.48$, and 25.45 was estimated to be $0.4133,0.2952$, and 0.2362 , respectively. The crystallite size diameter (D) of the $\mathrm{TiO}_{2}$ products has been calculated by Debye-Scherrer equation, $\mathrm{D}=0.9 \mathrm{k} / \beta \cos \theta$. Calculated crystalline domain sizes have been found to be 21, 30, and $38 \mathrm{~nm}$ for the samples S1, S0 and S1T0, respectively. It can be concluded that the employed Schiff-base ligand has a significant influence on crystalline size of the product so that in the presence of only ligand (sample S1T0) the calculated crystalline domain size increased to $38 \mathrm{~nm}$. It seems that applying both the ligand and TEOA (S1) has a different effect so that the particle size of S0 sample was smaller than S1T0. Preparation of $\mathrm{TiO}_{2}$ nanoparticles with tuneable size is of great interest for different applications. Here, it has been shown that different conditions lead to different crystalline size from ca. 21-38.

Fig. 1d shows the XRD patterns of sample T1prepared in time 2 days. All the peaks were labelled and can be indexed to JCPDS Card No. 71-1166, 88-1175, and 31-1827 show anatase, rutile, and impurities, respectively. The data for the full width at half maximum (FWHM) of sample T1 at $2 \theta=20.0148$ was estimated to be 0.1771 and the calculated crystalline domain size has been found to be $52 \mathrm{~nm}$ for sample $\mathrm{T} 1$.

FT-IR spectra have been demonstrated in Fig. 2a. Based on these spectra, $\operatorname{Salpn}\left(\mathrm{NO}_{2}\right)_{2}$ as a ligand has been synthesized successfully (Fig. 2a). The board peak on $3350 \mathrm{~cm}^{-1}$ was depended to $\mathrm{OH}$, the sharp peak on 1642 $\mathrm{cm}^{-1}$ was pertained to $\mathrm{C}=\mathrm{N}$; the peaks that were placed on $1514 \mathrm{~cm}^{-1}, 1342 \mathrm{~cm}^{-1}$ are attributed to symmetric and asymmetric stretching vibration of O-N-O and the peak that occurred in $2850 \mathrm{~cm}^{-1}$ is the result of $\mathrm{CH}_{2}$ in ligand structure. 
The surface conditioning of as-prepared $\mathrm{TiO}_{2}$ product was recorded using FT-IR spectrum in order to detect the residual organic compounds. Fig. 2b shows FT-IR spectrum of L1 sample. The absorption from 3000 to 3600 $\mathrm{cm}^{-1}$ can be assigned to the stretching vibration of the hydrogen-bonded $\mathrm{OH}$ groups of the adsorbed water. The absorption around $1628 \mathrm{~cm}^{-1}$ is due to the bending vibration of water molecules. The broad band below $950 \mathrm{~cm}^{-1}$ in the FT-IR spectrum belongs to the characteristic vibrations of the inorganic Ti-O-Ti network. The bands at around 2920 and $2850 \mathrm{~cm}^{-1}$ are assigned to the antisymmetric and symmetric $\mathrm{C}-\mathrm{H}$ stretching vibrations of hydrocarbon moiety.

EDX technique was used to determine the chemical composition of the products (Fig. 3). The EDX of the S1 sample has been shown in Fig. 3. Peaks associated with Ti and $\mathrm{O}$ are clearly observed and provide strong evidence that the nanocrystals are composed of only $\mathrm{TiO}_{2}$.

The morphology of the products has been examined by SEM images (Fig. 4, 5), respectively. Fig. 4 a-c show the SEM images of $\mathrm{TiO}_{2}$ nanoparticles treated at different times (T1, T2, and T3). Fig. 4a is the image of $\mathrm{TiO}_{2}$ nanoparticles treated by means of 2 days. This image shows that most particles are in inferior morphology and size. By increasing the time to 4 days, the particles disjoined and uniformity increased (Fig. 4b). But by increasing the time from 4 to 5 days, the particle agglomeration increased and large particles were produced (Fig. 4c). Thus, the optimum time was 4 days to prepare $\mathrm{TiO}_{2}$ nanoparticles (Fig. 4b).

In order to observe the size and morphology dependence of $\mathrm{TiO}_{2}$ products on the concentration of ligand and different metals, ligand ratios were tested (Fig. 5). The effect of ligand concentration on the surface morphologies of the obtained $\mathrm{TiO}_{2}$ products has been shown in Fig. 4, 5. Fig. 4b shows $\mathrm{TiO}_{2}$ samples $\mathrm{S} 1$ and Fig. 4a-d show $\mathrm{TiO}_{2}$ samples $\mathrm{S} 0, \mathrm{~S} 0.5, \mathrm{~S} 3$ and $\mathrm{S} 6$, respectively.

The absorption spectra of the as-prepared $\mathrm{TiO}_{2}$ powders were recorded and have been shown in Fig. 6. It shows that the absorption edges of different samples are slightly changing with increasing ligand concentration. The 
fundamental absorption edge in most semiconductors follows the exponential law. Using the absorption data, the band gap was estimated by Tauc's relationship:

$$
\alpha=\frac{a_{0}\left(h v-E_{\mathrm{g}}\right)^{\mathrm{n}}}{\mathrm{hu}}
$$

Where $\alpha$ is the absorption coefficient, ho is the photon energy, $\alpha_{0}$ and $\mathrm{h}$ are the constants, $\mathrm{E}_{\mathrm{g}}$ is the optical band gap of the material, and $n$ depends on the type of electronic transition and can be any value between $1 / 2$ and 3 . The energy gaps of the samples have been determined by the intercept of the plots of $(\alpha \text { hv })^{1 / 2}$ against hv to the energy axis (inset in Fig. 6). The energy gaps of the samples have been determined from the intercept of the plots of $(\alpha h v)^{1 / 2}$ against hv to the energy axis (inset in Fig. 6). The $E_{\mathrm{g}}$ values were calculated 2.82, 3, 3.05 and $3.09 \mathrm{eV}$ for the S0.5, S1, S3 and S6 samples, respectively. The energy gap calculated of the sample without Schiff-base ligand Salpn $\left(\mathrm{NO}_{2}\right)_{2}$ is $2.79 \mathrm{eV}$ (inset in Fig. 7). For the $\mathrm{TiO}_{2}$ anatase phase, values from 2.86 to $3.34 \mathrm{eV}$ have been reported in the literature and the differences have been attributed to variations in the stoichiometric of the synthesis, the impurities content, the crystalline size and the type of electronic transition. Here, it is observed that increasing the ligand concentration results in shifting the band gap to the higher values. The band gap shifting due to ligand concentration may refer to the size reduction of $\mathrm{TiO}_{2}$ nanoparticles with controlling the growth rate of nuclei with higher adsorption of ligands on the surface of $\mathrm{TiO}_{2}$ embryos.

\section{Conclusions}

In summary, $\mathrm{TiO}_{2}$ nanoparticles of different sizes and agglomeration levels were prepared by applying organic Schiff-base ligand (Salpn(NO2)2)) using the two step sol-gel method. It was revealed that the organic Schiff-base ligand as the complexing agent has a crucial role on the morphology of the products. In this study, for the first time, we utilized Schiff-base organic ligand, ( $\left.\mathrm{Salpn}\left(\mathrm{NO}_{2}\right) 2\right)$, as complexing agent in synthesis of $\mathrm{TiO}_{2}$ nanoparticles.

\section{Acknowledgment}


Authors are grateful to the council of Iran National Science Foundation and University of Kashan for supporting this work by Grant No (159271/742). 


\section{References}

[1] H.S. Jung, H. Shin, J.R. Kim, J.Y. Kim, K.S. Hong, J.K. Lee, Langmuir 20 (2004) 11732-7.

[2] Z. Yuan, J. Zhang, B. Li, J. Li, Thin Solid Films 515 (2007) 7091-5.

[3] K.T. Lim, H.S. Hwang, S.S. Hong, C. Park, W. Ryoo, K.P. Johnston, Stud. Surf. Sci. Catal. 153 (2004) 569-

72.

[4] P.E. Meskin, V.K. Ivanov, A.E. Barantchikov, B.R. Churagulov, Y.D. Tretyakov, Ultrason. Sonochem. 13 (2006) 47-53.

[5] E. Gressel-Michel, D. Chaumont, D. Stuerga, J. Colloid Interface Sci. 285 (2005) 674-679.

[6] P.-T. Hsiao, M.-D. Lu, Y.-L. Tung, H. Teng, J. Phys. Chem. C 114(2010) 15625-32.

[7] T. Sugimoto, K. Okada, H. Itoh, J. Colloid Interface Sci. 193 (1997)140-3.

[8] T. Sugimoto, X. Zhou, A. Muramatzu, J. Colloid Interface Sci. 252 (2002) 339-46.

[9] T. Sugimoto, X. Zhou, A. Muramatzu, J. Colloid Interface Sci. 259 (2003) 43-52.

[10] K. Kanie, T. Sugimoto, J. Am. Chem. Soc. 125 (2003) 10518-9.

[11] M. Froba, O. Muth, A. Relier, Solid State Ionics 101-103 (1997) 249-53.

[12] T. Nishide, F. Mizukami, Thin Solid Films 353 (1999) 67-71.

[13] Z. Hens, I. Moreels, B. Fritzinger, J.C. Martins, Comprehensive Nanoscience and Technology 5 (2011) 2149.

[14] Z. Shahri, M. Bazarganipour, M. Salavati-Niasari, Superlattices Microstruct. 63 (2013) 258-266.

[15] M. Salavati-Niasari, B. Shoshtari-Yeganeh, M. Bazarganipour, Superlattices Microstruct. 58 (2013) $20-30$.

[16] T. Gholami, M. Salavati-Niasari, M. Bazarganipour, E. Noori, Superlattices Microstruct. 61 (2013) $33-41$.

[17] M. Salavati-Niasari, M. Bazarganipour, J. Clust. Sci. 23 (2012) 503-9.

[18] E. Noori, M. Bazarganipour, M. Salavati-Niasari, T. Gholami, J. Clust. Sci. 24 (2013) 1171-1180

[19] M. Goudarzi, M. Bazarganipour, M. Salavati-Niasari, RSC Adv. 4 (2014) 46517- 46520. 
[20] S. Mandizadeh, M. Bazarganipour, M. Salavati-Niasari, Ceramics International, 40 (2014) 15685-15691.

[21] S. Gholamrezaei, M. Salavati-Niasari, M. Bazarganipour, M. Panahi-Kalamuei, S. Bagheri, Advanced Powder Technology, 25 (2014) 1585-1592.

[22] G. Li, L. Chen, J. Bao, T. Li, F. Mei, Appl. Catal. A: Gen. 346 (2008) 134-9.

[23] S. Lee, I.-S. Cho, J.H. Lee, D.H. Kim, D.W. Kim, J.Y. Kim, H. Shin, J.-K. Lee, H.S. Jung,N.-G. Park, K.

Kim, M.J. Ko, K.S. Hong, Chem. Mater. 22 (2010) 1958-65.

[24] T. Sugimoto, X. Zhou, A. Muramatsu, J. Colloid Interface Sci. 259 (2003) 53-61.

[25] A. Sobhani, M. Salavati-Niasari, Ceram. Int. 40 (2014) 8173-8182.

[26] A. Sobhani, M. Salavati-Niasari, J. Alloys Compd. 617( 2014) 93-101.

[27] A. Sobhani, M. Salavati-Niasari, J. Alloys Compd. 625 (2015) 26-33.

[28] F. Ansari, A. Sobhani, M. Salavati-Niasari, RSC Adv. 4 (2014) 63946-63950. 


\section{Figure captions:}

Fig. 1. XRD patterns of the $\mathrm{TiO}_{2}$ samples: (a) S1, (b) S0, (c) S1T0 and (d) T1.

Fig 2. FT-IR spectra of (a) Schiff base ligand $\left(\mathrm{Salpn}\left(\mathrm{NO}_{2}\right)_{2}\right.$, (b) $\mathrm{TiO}_{2}$ nanoparticles(S1).

Fig 3 EDX pattern of $\mathrm{TiO}_{2}$ nanoparticles (S1).

Fig. 4 SEM images of $\mathrm{TiO}_{2}$ prepared using two-step sol-gel method in the time different: (a) T1, (b) T2(S1) and

(c) T3.

Fig 5. SEM images of the $\mathrm{TiO}_{2}$ samples: (a) S0, (b) S0.5, (c) S3 and (d) S6.

Fig. 6. UV-Vis diffuse absorption spectra of the $\mathrm{TiO}_{2}$ samples: (a) S0.5, (b) S1, (c) S3 and (d) S6 the inset shows corresponding linear portion of the plots of $(\alpha h v)^{1 / 2}$ against $\mathrm{E}(\mathrm{eV})$.

Fig. 7. UV-Vis diffuse absorption spectra of the $\mathrm{TiO}_{2}$ samples: $\mathrm{S} 0$ the inset shows corresponding linear portion of the plots of $(\alpha h v)^{1 / 2}$ against $\mathrm{E}(\mathrm{eV})$.

Table 1. Different samples prepared in various aspect ratios.

Table 2. Different samples prepared in various times.

Scheme 1. Effect of differenttimes on morphology of $\mathrm{TiO}_{2}$ nanoparticles. 


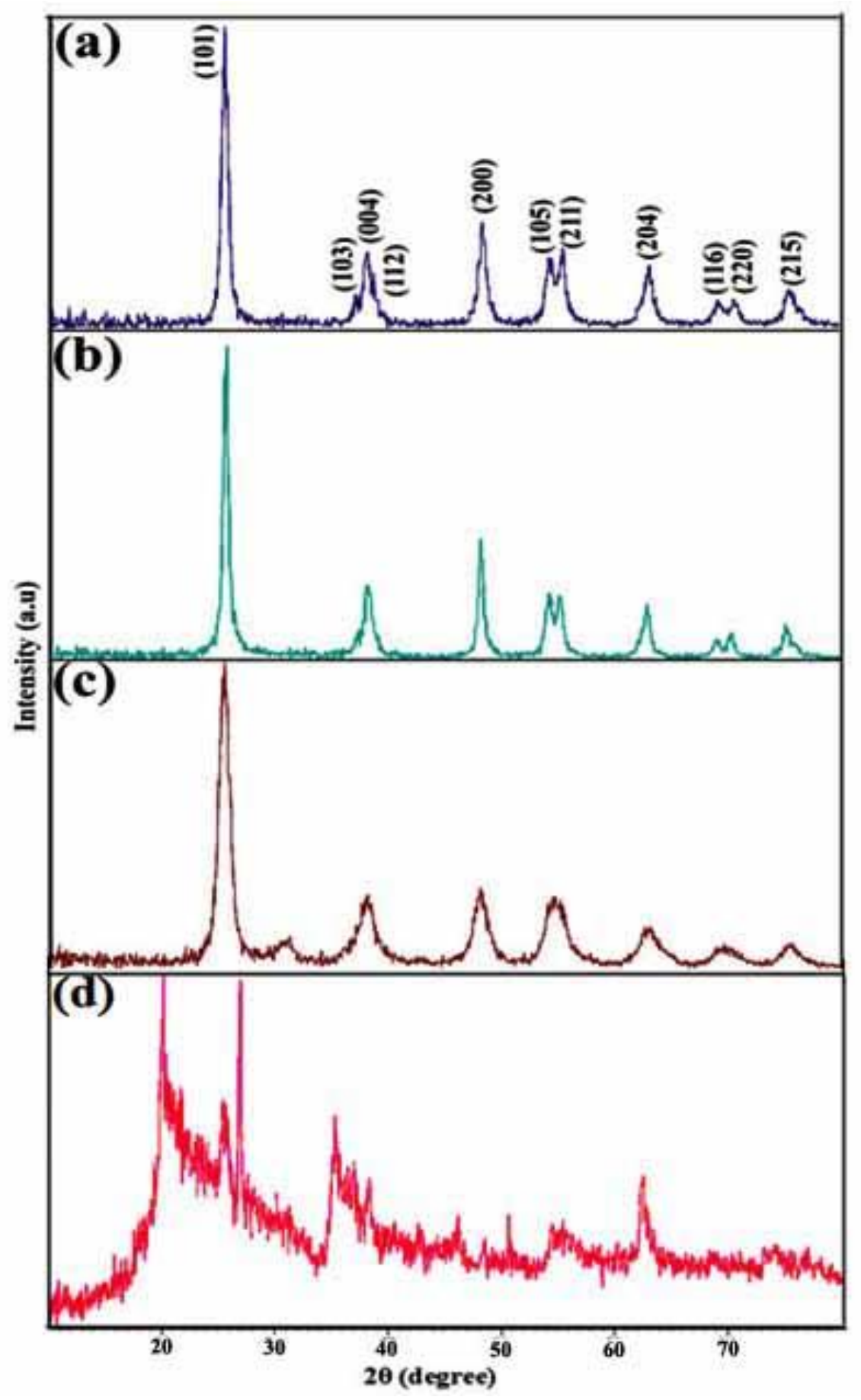

Fig. 1. XRD patterns of the $\mathrm{TiO}_{2}$ samples: (a) S1, (b) S0, (c) S1T0 and (d) T1. 


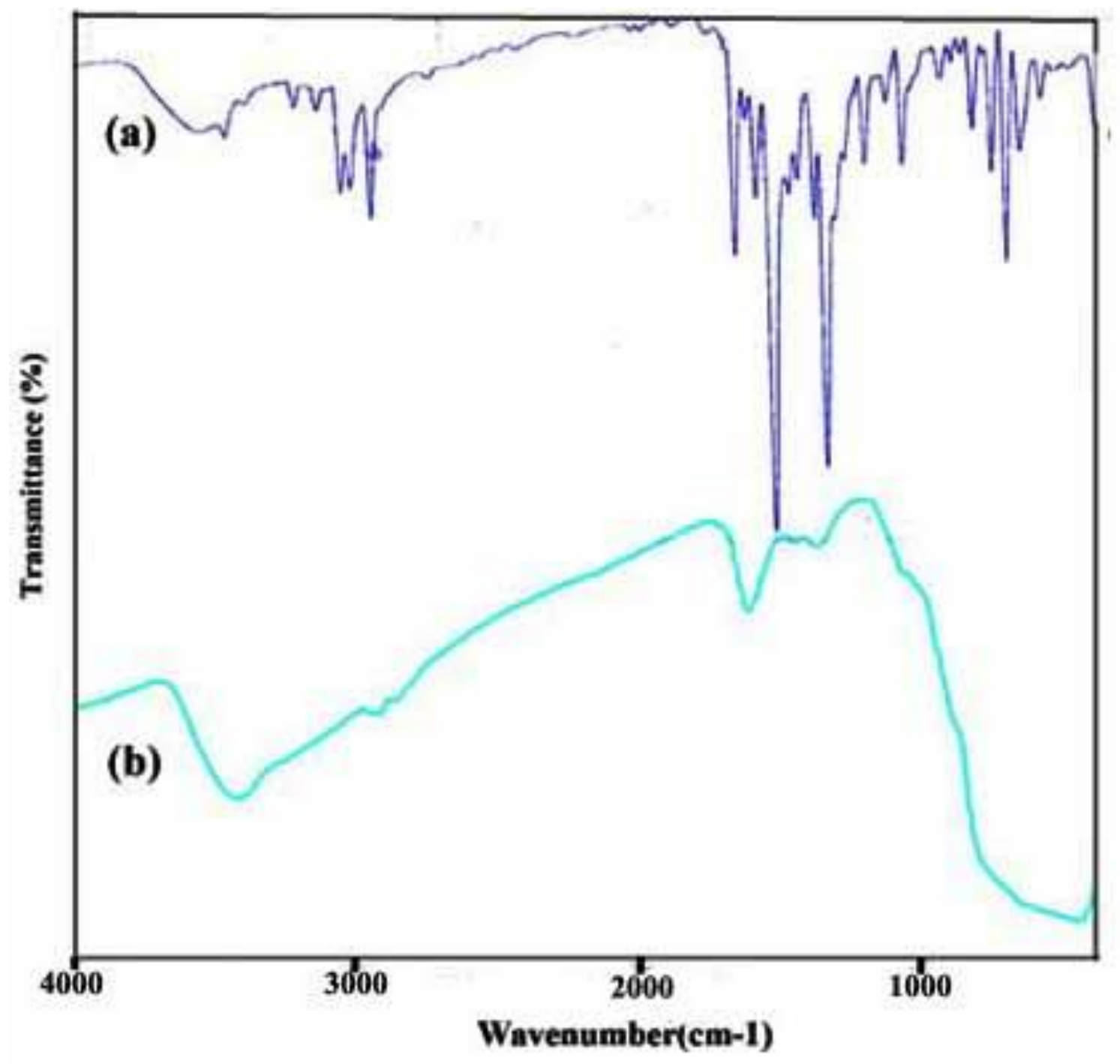

Fig. 2. FT-IR spectra of (a) Schiff base ligand ( $\mathrm{Salpn}\left(\mathrm{NO}_{2}\right)_{2}$, (b) $\mathrm{TiO}_{2}$ nanoparticles (S1). 


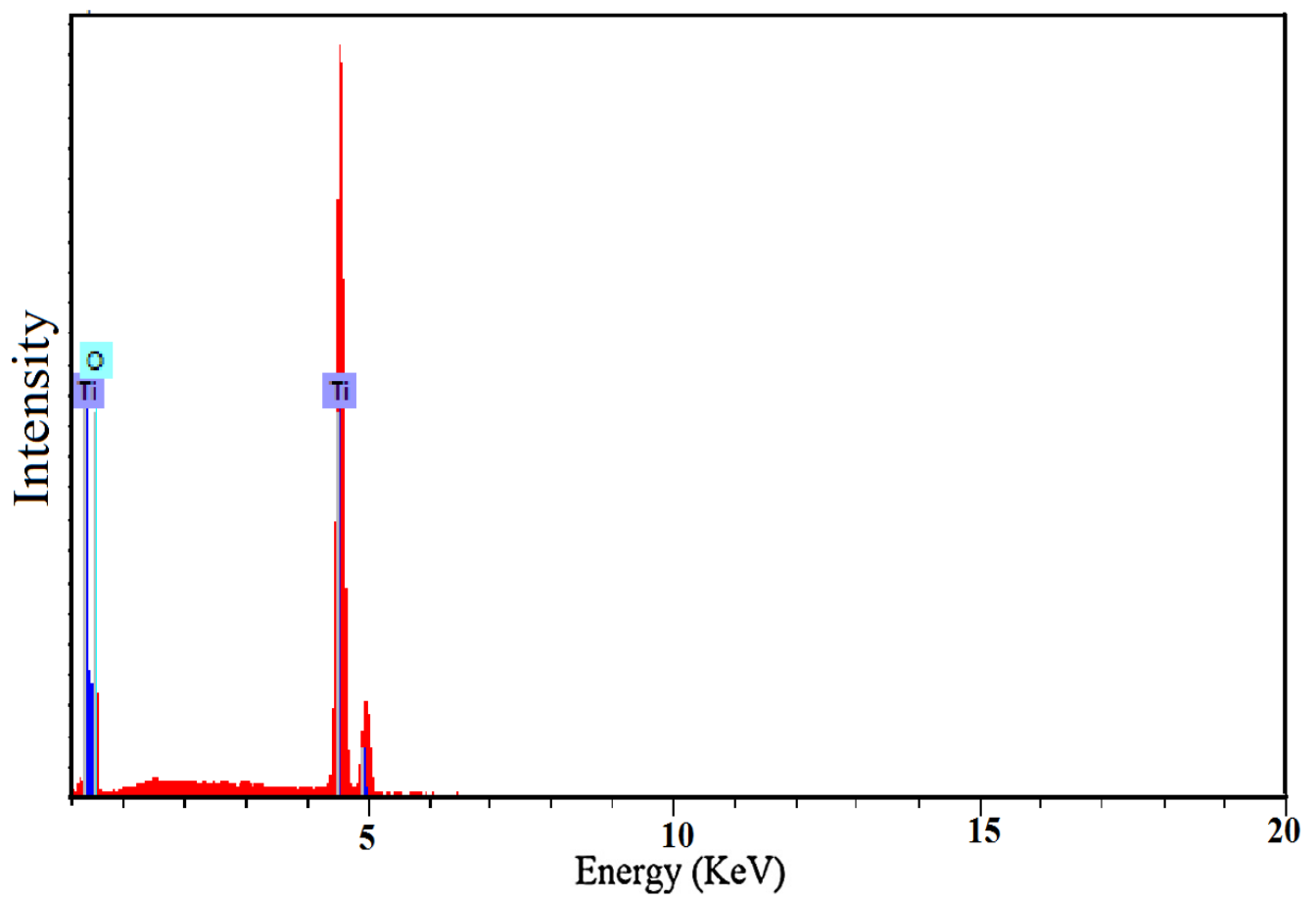

Fig. 3. EDX pattern of $\mathrm{TiO}_{2}$ nanoparticles (S1). 


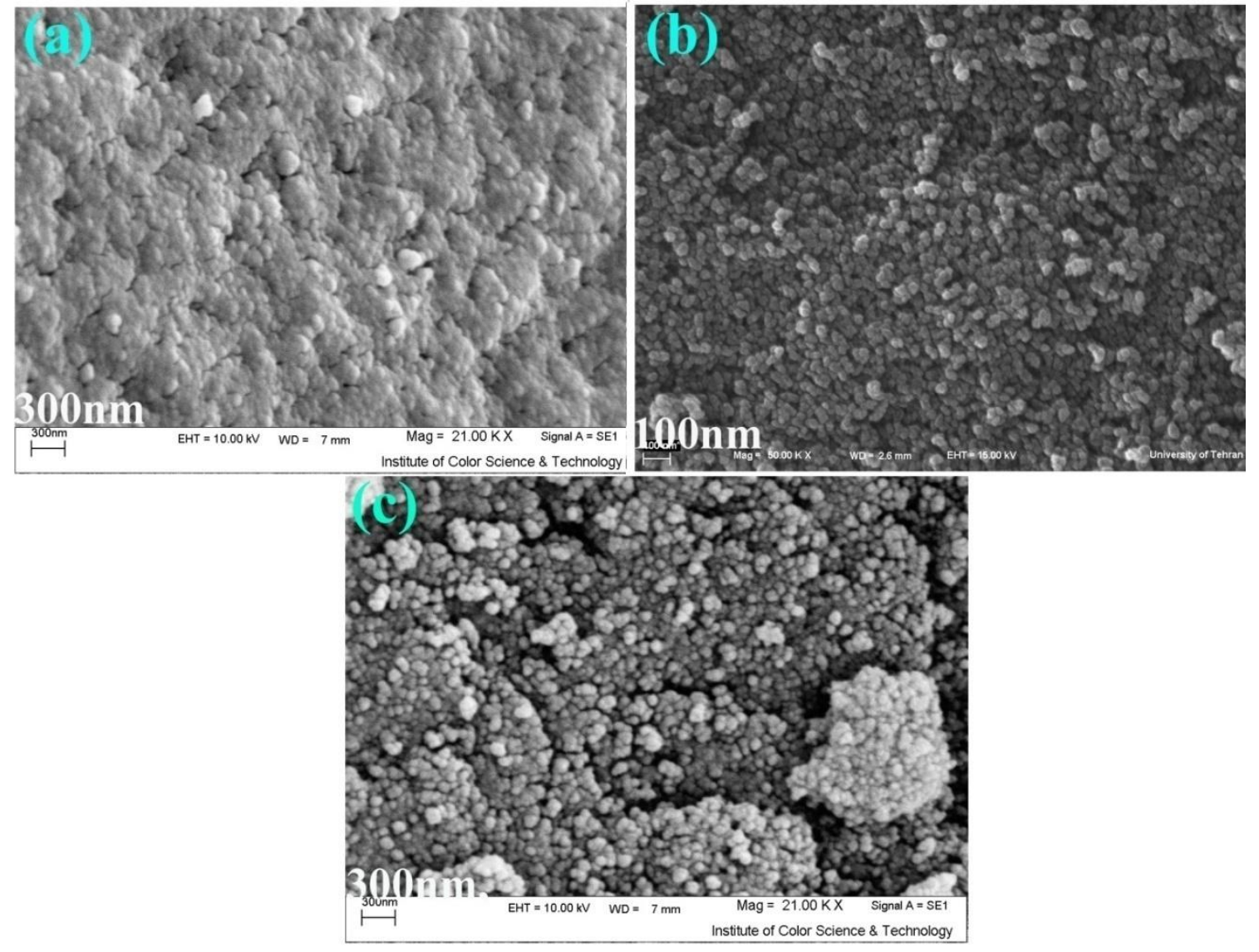

Fig. 4. SEM images of $\mathrm{TiO}_{2}$ prepared using two-step sol-gel method in the time different: (a) T1, (b) T2 (S1) and (c) T3. 


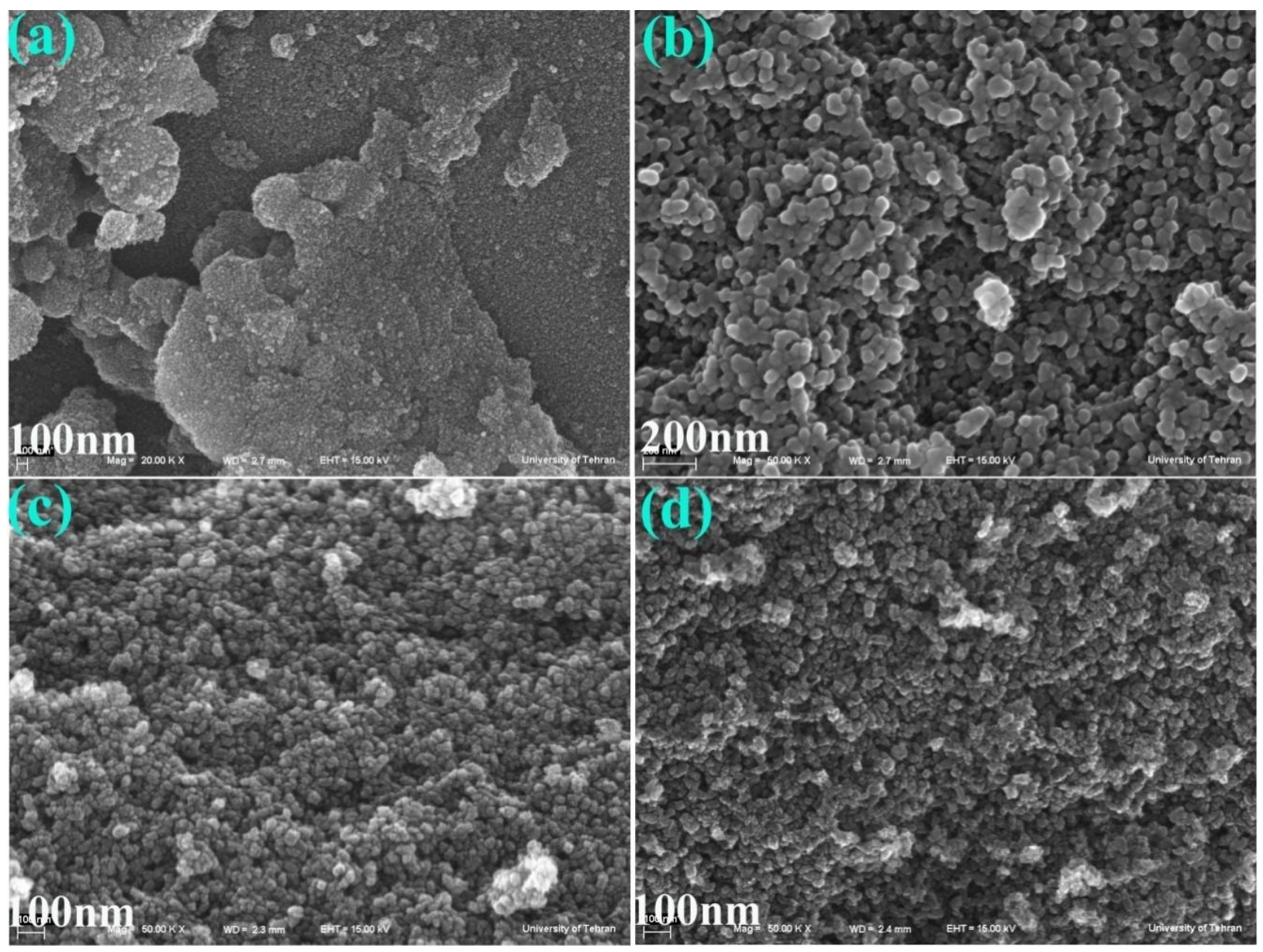

Fig 5. SEM images of the $\mathrm{TiO}_{2}$ samples: (a) S0, (b) S0.5, (c) S3 and (d) S6. 


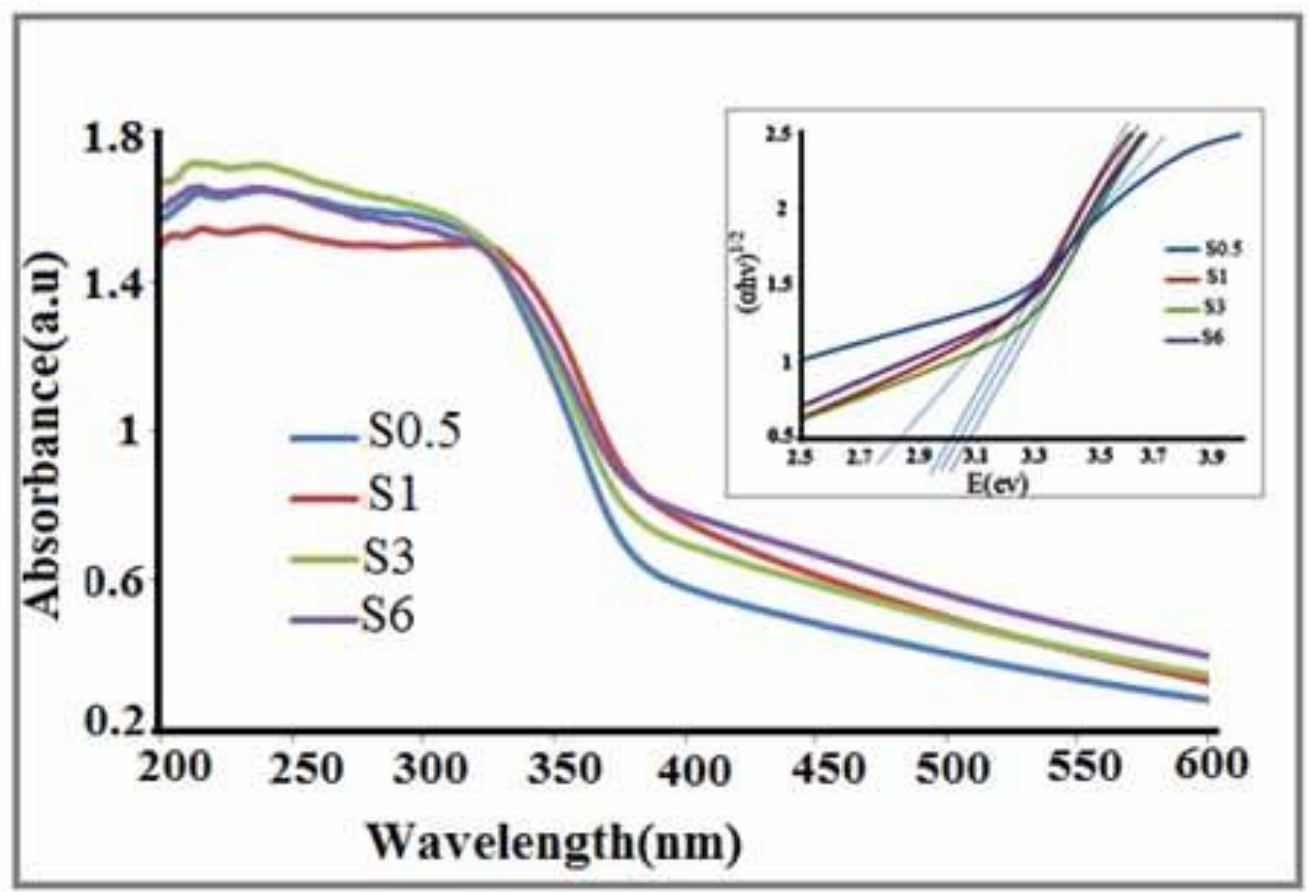

Fig. 6. UV-Vis diffuse absorption spectra of the $\mathrm{TiO}_{2}$ samples: (a) S0.5, (b) S1, (c) S3 and (d) S6 the inset shows corresponding linear portion of the plots of $(\alpha \mathrm{hv})^{1 / 2}$ against $\mathrm{E}(\mathrm{eV})$. 


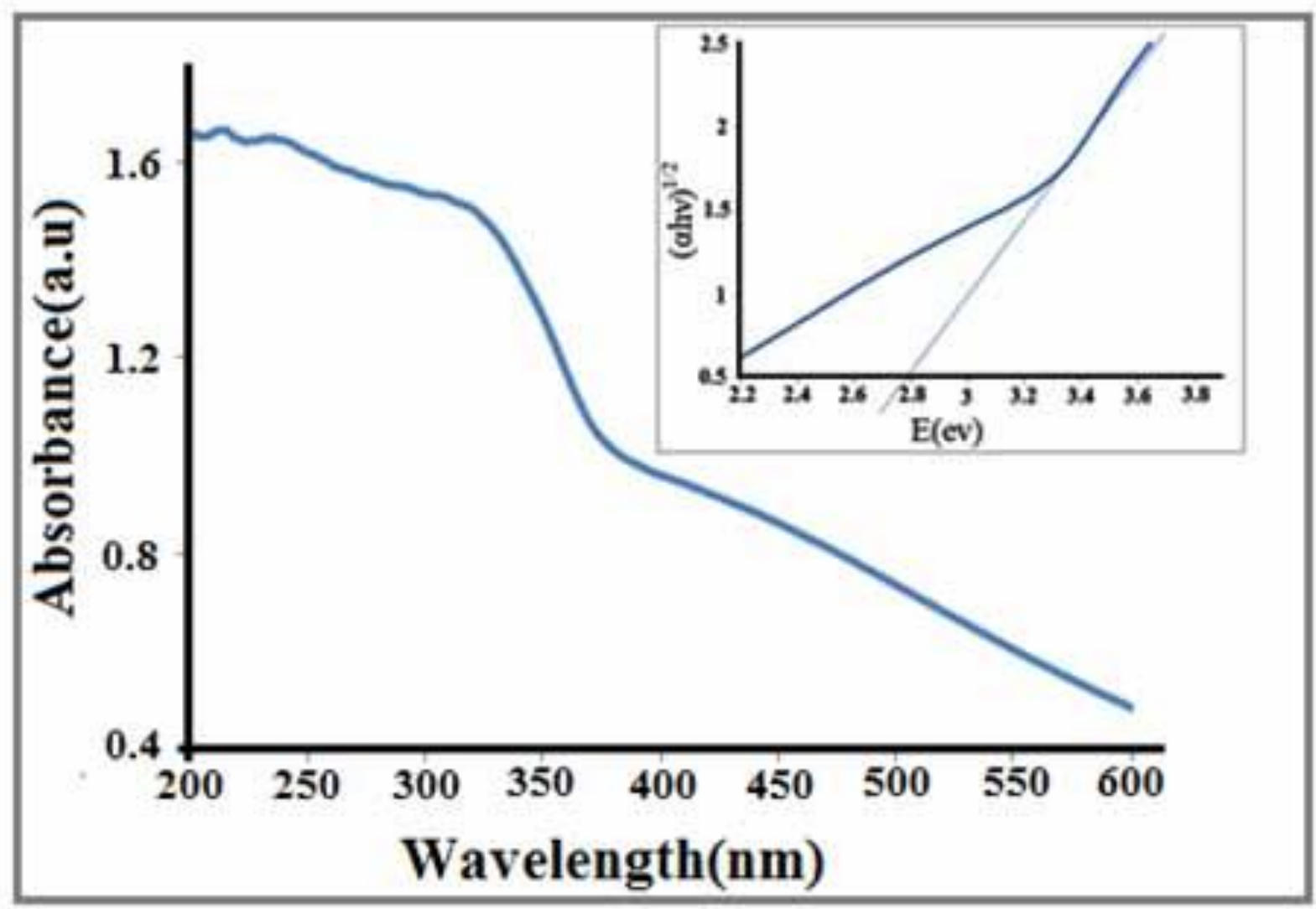

Fig. 7. UV-Vis diffuse absorption spectra of the $\mathrm{TiO}_{2}$ samples: $\mathrm{S} 0$ the inset shows corresponding linear portion of the plots of $(\alpha h v)^{1 / 2}$ against $\mathrm{E}(\mathrm{eV})$. 
Table 1. Different samples prepared in various aspect ratios.

\begin{tabular}{ccc}
\hline Sample abbreviation & $\mathrm{Ti}^{+4}: \mathrm{S}$ & $\mathrm{Ti}^{+4}:$ TEOA \\
\hline S1T0 & $1: 1$ & $1: 0$ \\
S0 & $1: 0$ & $1: 2$ \\
S0.5 & $1: 0.5$ & $1: 2$ \\
S1 & $1: 1$ & $1: 2$ \\
S3 & $1: 3$ & $1: 2$ \\
S6 & $1: 6$ & $1: 2$ \\
\hline
\end{tabular}

S: Schiff-base ligand

T: TEOA 
Table 2. Different samples prepared in various times.

\begin{tabular}{ccc}
\hline Sample abbreviation & \multicolumn{2}{c}{ Time, Temperature } \\
\hline $\mathrm{T}_{1}$ & $12 \mathrm{~h}, 100^{\circ} \mathrm{C}$ & $36 \mathrm{~h}, 140^{\circ} \mathrm{C}$ \\
$\mathrm{T}_{2}$ & $24 \mathrm{~h}, 100^{\circ} \mathrm{C}$ & $72 \mathrm{~h}, 140^{\circ} \mathrm{C}$ \\
$\mathrm{T}_{3}$ & $30 \mathrm{~h}, 100^{\circ} \mathrm{C}$ & $90 \mathrm{~h}, 140^{\circ} \mathrm{C}$ \\
\hline
\end{tabular}




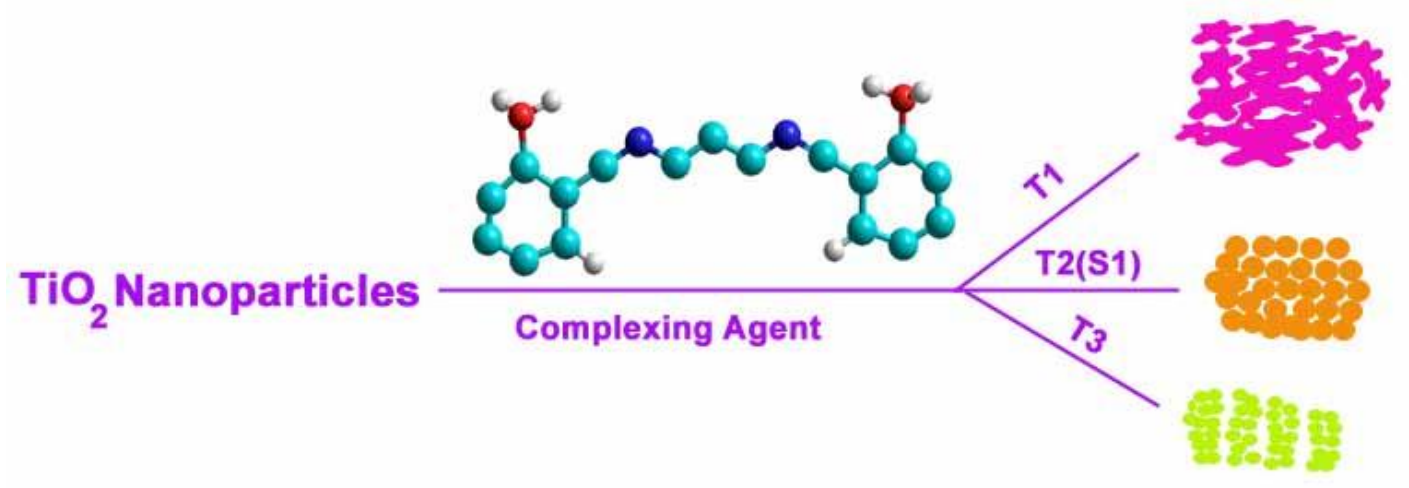

Scheme 1. Effect of different times on morphology of $\mathrm{TiO}_{2}$ nanoparticles. 
Graphical Abstract

$\mathrm{TiO}_{2}$ Nanoparticles

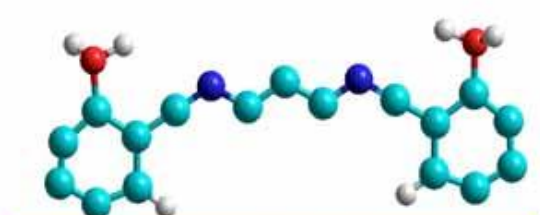

Complexing Agent

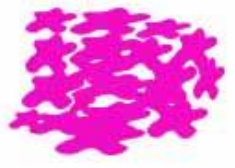

T2(S1)

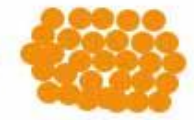

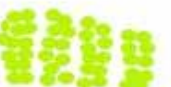

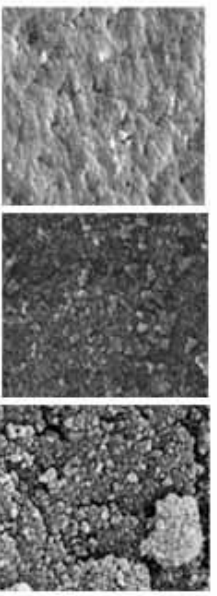

